\title{
Fiber-to-chip light coupling using a graded-index lensed fiber collimator
}

\author{
Siegfried Bondarenko, ${ }^{a}$ Marcel Hülsemann, ${ }^{a}$ Andreas Mai,,${ }^{a, b}$ and \\ Patrick Steglich $\odot^{\mathrm{a}, \mathrm{b}, *}$ \\ ${ }^{a}$ Technische Hochschule Wildau, Wildau, Germany \\ bIHP-Leibniz-Institut für innovative Mikroelektronik, Frankfurt(Oder), Germany
}

\begin{abstract}
Fiber-to-chip light coupling using a graded-index (GRIN) fiber collimator is investigated. Our experiments with grating couplers and strip waveguides fabricated in a photonic integrated circuit technology reveal that the peak coupling efficiency of a GRIN fiber collimator is $7.8 \mathrm{~dB}$ lower than that of a single-mode fiber. However, the 3-dB alignment tolerance is improved by a factor of about 5.7 giving rise to pluggable sensor solutions. This work opens a path toward a cost-effective and portable sensor platform based on pluggable photonic biosensors using GRIN fiber collimators. (C) 2021 Society of Photo-Optical Instrumentation Engineers (SPIE) [DOI: 10.1117/1.OE.60.1.014105]
\end{abstract}

Keywords: integrated photonics; fiber coupling; grating coupler; graded index-lensed fiber; silicon photonics.

Paper 20201209 received Oct. 11, 2020; accepted for publication Jan. 13, 2021; published online Jan. 29, 2021.

\section{Introduction}

Similar to microelectronics, photonics is moving toward miniaturization with increased functionality, scalability, and low costs. Photonic integrated circuits (PICs) are analogous to electronic integrated circuits, where instead of electrons, photons take over functions such as signal processing, ${ }^{1}$ amplification, ${ }^{2}$ modulation, ${ }^{3}$ detection, ${ }^{4}$ sensing,${ }^{5}$ and logical operations. ${ }^{6}$ As PICs are manufactured on silicon-based substrates like their electronic counterparts, they fall under the umbrella of silicon photonics, meaning matured complementary-metal oxide semiconductor technologies can be employed for fabrication. ${ }^{7}$ The recent introduction of silicon photonic devices in life sciences, and specifically in environmental monitoring, food safety and biomedical screening, offers highly promising perspectives. ${ }^{8,9}$ One general bottle-neck of silicon-based PICs is the lack of cost-effective integrated light sources.

To tackle this general issue, several solutions based on fiber coupling techniques were developed during the last few years. ${ }^{10}$ The solutions can be categorized in butt coupling ${ }^{11}$ and grating coupling. ${ }^{12}$ Butt coupling is typically utilized for applications in telecommunication since it provides low coupling-loss, large spectral bandwidth, and low-sensitivity to polarization. ${ }^{13}$ The main drawbacks are its tight alignment tolerances (sub-micron level) and the need of sophisticated packaging procedures. On the other hand, an advantage of grating couplers over butt couplers is the possibility of wafer-level testing. ${ }^{14}$

Silicon-based PICs for biosensing require lower light intensity compared to PICs for highspeed signal processing. Current research is focused on the optimization of light coupling in terms of optical insertion losses but also on packaging solutions for pluggable light coupling. The latter is necessary for future disposable biosensor solutions. One approach is the use of micro-lenses, as presented by Scarcella et al. ${ }^{15}$ In this case, a pair of micro-lens arrays is employed to obtain grating-coupling with an insertion-loss of $1.7 \mathrm{~dB}$, and a $1 \mathrm{~dB}$ alignment tolerance of $\pm 30 \mu \mathrm{m}$. Another solution is based on 3D nanoprinting of free-form coupling elements, as presented by Dietrich et al. ${ }^{16}$

*Address all correspondence to Patrick Steglich, steglich@ihp-microelectronics.com

0091-3286/2021/\$28.00 (C) 2021 SPIE

Optical Engineering

014105-1

January $2021 \cdot$ Vol. 60(1) 


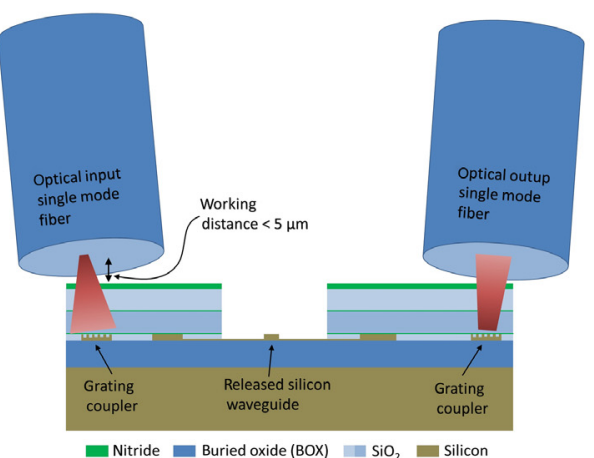

(a)

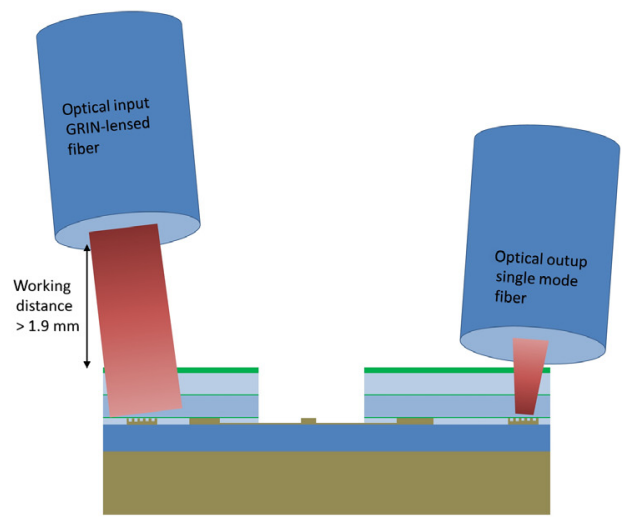

(b)

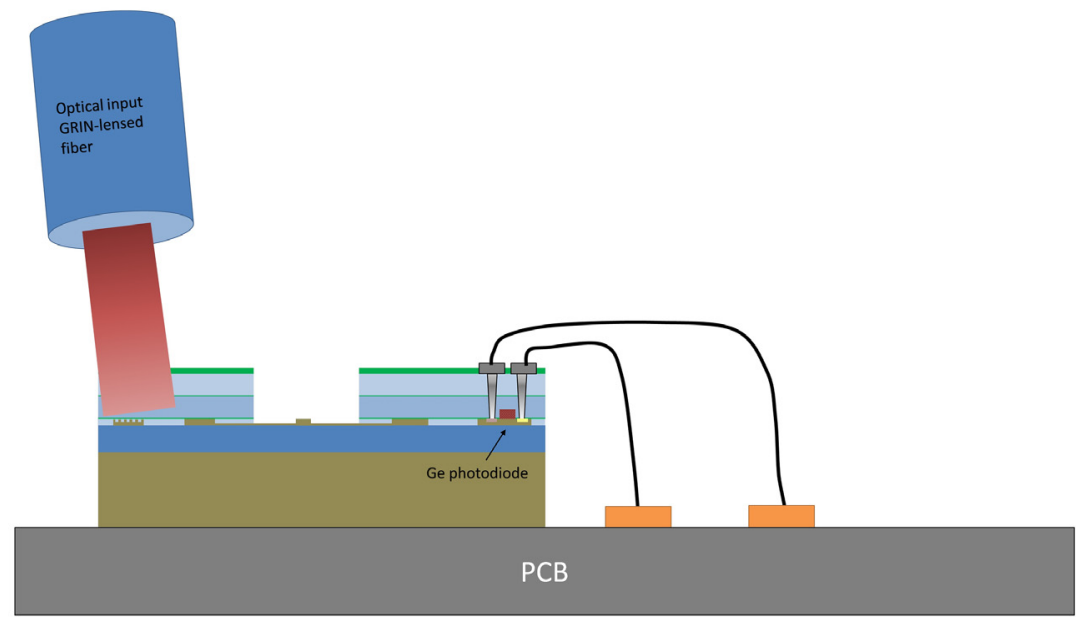

(c)

Fig. 1 (a) Schematic cross section of a photonic chip. The light is usually coupled via SMFs and grating coupler into the chip. The narrow working distance makes a practical use for pluggable chips challenging. (b) Using a GRIN-lensed fiber allows a much larger working distance. Envisioned photonic sensor chip packaged on a PCB. (c) Here, the photodiode is integrated on chip and connected to the PCB through wire bonding.

However, the most common approach is based on fiber-to-chip coupling using grating coupler, as shown in Fig. 1(a). The advantage that makes it most attractive for practical use is the fact that the fiber can be reused if it is not packaged with the chip, which is significantly more costeffective than the aforementioned solutions. Therefore, this approach is preferred for experiments in laboratories. However, due to the narrow alignment tolerance and working distance, it is not expedient to use this approach for practical applications in the field of biosensing, as for example point-of-care-diagnostics, which hinders the development of cost-effective, pluggable photonic biosensors with reusable optical fibers. A comprehensive overview of fiber-to-chip coupling strategies can be found in Ref. 10.

In this work, we investigate on a straightforward fiber-to-chip light coupling solution to overcome this issue without fiber packaging. We propose the use of a graded-index (GRIN)-lensed fiber collimator to obtain a large fiber-to-chip distance and an improved alignment tolerance, which is required for pluggable photonic biosensors. This allows a higher alignment tolerance and working distance at the same time [Fig. 1(b)]. Following the proposed approach, a pluggable biosensor module based on a photonic chip bonded on a printed circuit board (PCB) can be envisioned as application of GRIN-lensed fiber coupling. For the first time, we prove the viability of GRIN-lensed fibers for an alignment tolerant coupling and show that the coupling efficiency is appropriated to measure the spectra of a ring resonator, which is typically used as photonic biosensor. So far, GRIN-lensed fibers are fabricated on the tip of a single-mode fiber 
$(\mathrm{SMF})^{17}$ or multi-mode GRIN-lensed fibers are used for butt coupling. ${ }^{18}$ However, this work investigates for the first time, to our best knowledge, single-mode GRIN-lensed fiber in combination with on-chip grating coupler.

\section{Theory}

A potential solution to the limited alignment tolerances of SMF is the replacement with GRINlensed fibers, which are used for fiber-to-fiber imaging, ${ }^{19}$ coupling laser diode light into fibers, ${ }^{20}$ and fiber-to-chip (detector) coupling. ${ }^{21}$ With the ability for light focusing and collimation and beam shaping, GRIN-lensed fibers are promising candidates for coupling light into pluggable sensor systems. A schematic of a GRIN lens attached to an optical fiber is shown in Fig. 2.

As the name suggests, a GRIN lens comprises a graded refractive index profile along the radius $r$ of the lens, enabling self-focusing within the lens. Manufacturing of the graded refractive index profile is performed by, e.g., silver ion exchange. ${ }^{22}$ An advantage of GRIN lenses over conventional spherical lenses is that the two lens surfaces are entirely plane, which enables an easy assembly and a small form factor. With lens diameters as small as $200 \mu \mathrm{m}$ it is possible to directly mount the GRIN lens to the endface of a typical $125 \mu \mathrm{m}$ SMF. The input plane of the GRIN lens and the endface (objective side) of the SMF are in direct contact. The light from the SMF core propagates through the objective focusing point at distance $s$ into the GRIN lens where it diverges, finally reaching its focal length $f$. In this example, the light rays become quasicollimated and are focused to a spot at the working distance $w$, where the beam waist $2 \omega_{0}$ (image side) can be found.

The change in refractive index $n$ along the lens radius $r$ is given by the equation

$$
n(r)=n_{0} \operatorname{sech}(g r),
$$

where $n_{0}$ is the refractive index at the center of the profile (optical axis) and $g$ is the refractive gradient constant (in $\mathrm{mm}^{-1}$ ). The hyperbolic secant function in Eq. (1) can be approximated as

$$
n(r) \approx n_{0}\left(1-\frac{g^{2}}{2} r^{2}\right)
$$

The lens length can be calculated as

$$
z_{l}=\frac{2 \pi}{g} P
$$

where $P$ is the pitch and one of the most important parameters of a GRIN lens, as it directly determines the working principle of the imaging system. The pitch $P$ expresses the number of

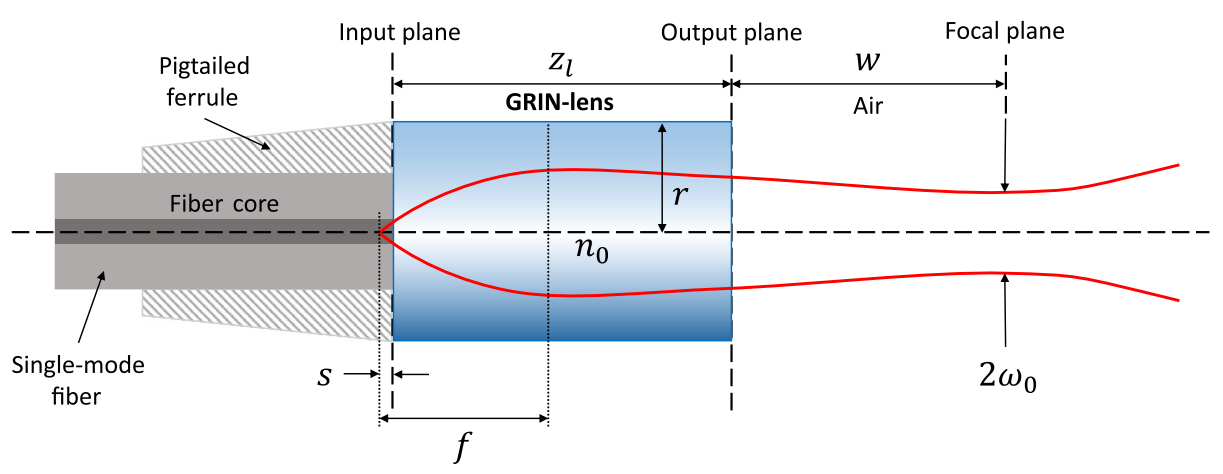

Fig. 2 Schematic cross section of a GRIN-lensed fiber and denotation of its parameters. The blue color gradient represents the graded refractive index profile of the lens. The red lines represent the marginal light rays. Light from the SMF enters the input plane of the GRIN-lens, where it diverges and is either collimated or focused behind the output plane, depending on the lens design. Here, the light is focused to a spot of diameter $2 \omega_{0}$ at the working distance $w$. 
continuous cosine ray traces along the lens length, which form due to the radial index profile. The length of a single cosine period $z_{P}$ is based on the relation $z_{P}=2 \pi / g$. Integrals of the pitch $(P=1,2,3, \ldots)$ lead to a 1:1 image reproduction, whereas a half pitch $(P=0.50)$ lens returns an inverted image and a quarter pitch lens $(P=0.25)$ collimates a point source at infinity. Pitches in between those values exist as well, for instance, the GRIN lens used in this work has a pitch of $\approx 0.27$, resulting in focusing at a relatively long working distance (as implied in Fig. 2).

\section{Experimental Setup}

A fiber-to-grating coupling setup is employed to measure the optical losses as a function of the fiber position. Figure 3 shows a schematic representation of the setup. The light of a tunable laser is coupled to either the single-mode or GRIN-lensed fiber, which is aligned with respect to the on-chip grating coupler. The coupling angle is $\theta=14 \mathrm{deg}$ [see also Fig. 4(b)]. The tunable laser covers the telecommunication C-band wavelength range and offers a wavelength accuracy of $\pm 5 \mathrm{pm}$, with output powers of up to $10 \mathrm{dBm}$. However, we used in the following experiments a fixed wavelength of $1550 \mathrm{~nm}$, at which the grating coupler have their maximum coupling efficiency. An optical isolator and polarization controller follow the outputs of both light sources,

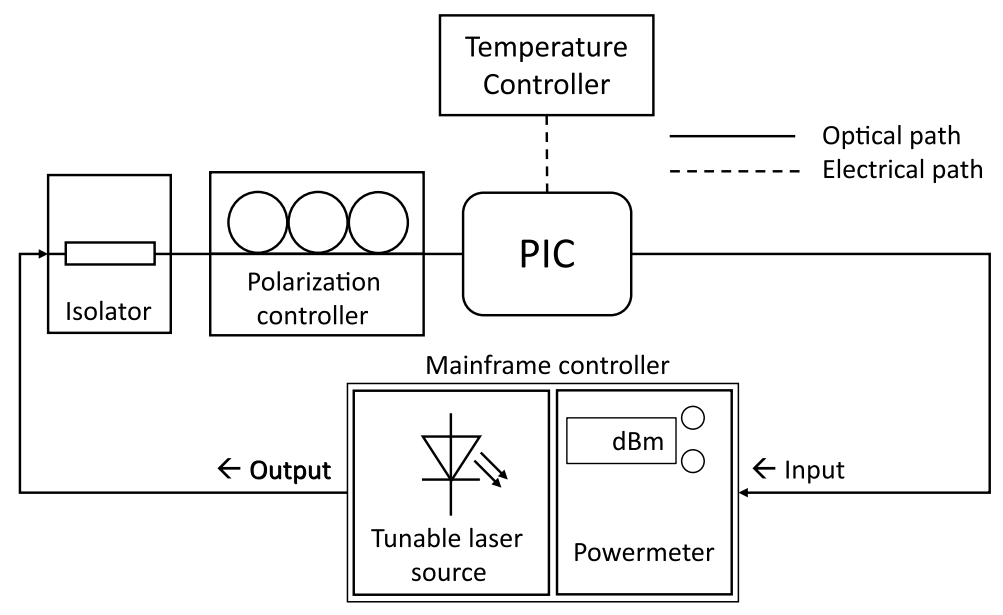

Fig. 3 Schematic of the high-precision setup for fiber-to-chip light coupling.

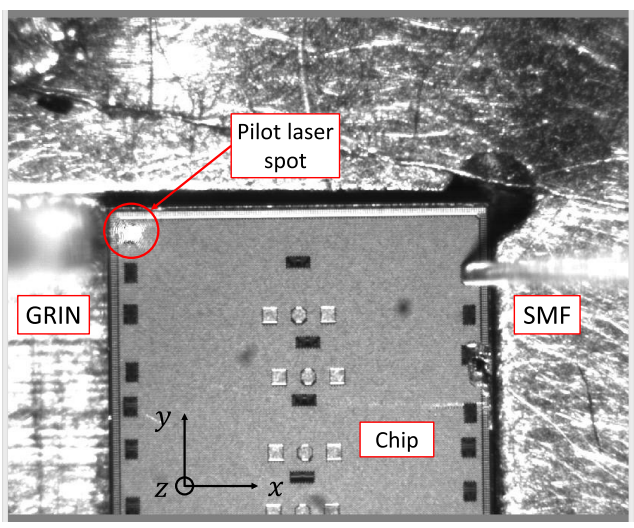

(a)

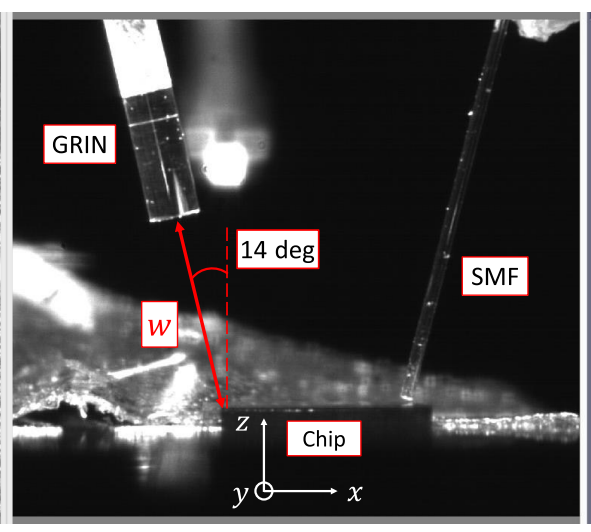

(b)

Fig. 4 (a) Top view and (b) side view of the optical coupling procedure. A pilot laser projects a visible light spot through the GRIN-lensed fiber on the input grating of the photonic chip to adjust the optimal fiber position and to find the working distance $w$. The coordinate system is included in each view. 
Table 1 Parameters of the custom ordered GRIN-lensed fiber configuration.

\begin{tabular}{llc}
\hline \hline Symbol & \multicolumn{1}{c}{ Parameter } & Value \\
\hline$P$ & Pitch & $\approx 0.27$ \\
$r$ & Lens radius & $0.25 \mathrm{~mm}$ \\
$z_{l}$ & Lens length & $1.26 \mathrm{~mm}$ \\
$f$ & Focal length & $0.46 \mathrm{~mm}$ \\
$s$ & Focus distance & $\approx 0 \mathrm{~mm}$ \\
$g$ & Gradient constant & $1.349 \mathrm{~mm}^{-1}$ \\
$n_{0}$ & Center refractive index & 1.616 \\
\hline \hline
\end{tabular}

Table 2 Most important differences between an SMF and the custom ordered GRIN-lensed fiber. Parameters taken from the respective data sheets of the manufacturers.

\begin{tabular}{lccc}
\hline \hline Fiber type & MFD $(\mu \mathrm{m})$ & $\begin{array}{c}\text { Working } \\
\text { distance } w(\mathrm{~mm})\end{array}$ & $\begin{array}{c}\text { Numerical } \\
\text { aperture }\end{array}$ \\
\hline SMF & 10.4 & 0.005 & 0.14 \\
GRIN-lensed fiber & 60.0 & 1.9 & 0.50 \\
\hline \hline
\end{tabular}

minimizing reflections and optimizing the polarization for the on-chip grating couplers of the PIC.

Here, the SMF is a commercially available Thorlabs P1-SMF28E-FC, whereas the GRINlensed fiber is a custom order by GRINTech GmbH. The latter consists of a Corning SMF28 with ferrule connector/angled physical contact (FC/APC) connector on one side, where the other side ends with an attached GRIN lens. A pigtailed (tapered) hollow glass ferrule is pushed over the spliced fiber, its endface is polished and the GRIN lens attached by UV-curable, transparent adhesive. The glass ferrule widens the diameter of the SMF to that of the GRIN lens and acts as a kink protection (see Fig. 2). Table 1 summarizes the configuration of the custom GRINlensed fiber.

Table 2 gives the most important differences between a standard SMF and a GRIN-lensed fiber. The mode field diameter (MFD) of the GRIN-lensed fiber is almost six times larger than that of an SMF. Considering the surface area of the on-chip grating couplers of the chip measure $15 \times 10 \mu \mathrm{m}$, it becomes evident that a larger MFD offers a higher coverage and thereby a possibly higher coupling tolerance. With a numerical aperture of 0.50 compared to 0.14 of the SMF, the GRIN lens allows for a stronger widening of the incident beam until collimation. A benefit of the large working distance of $1.9 \mathrm{~mm}$ is that it allows for more space between the housing and the pluggable module, improving the clearance during the plug and play action. That would not be possible with an SMF, which requires almost direct contact with the chip surface.

\section{Results}

In this experiment, we investigated the coupling efficiency of a GRIN-lensed fiber compared to that of an SMF. A photonic chip is fabricated in a PIC technology using 200-mm silicon-oninsulator wafer. A detailed description of the chip fabrication can be found in our prior work. ${ }^{23}$ Both fiber types were tested for the input grating, one at a time, while the light from the output grating was received by an SMF, which was fixed in position. A strip waveguide and an S-band is used between both grating coupler. The grating coupler and the employed silicon strip waveguides are acting as mode-filter because only the first TE mode is guided. The short waveguide 
length of $1.8 \mathrm{~mm}$ makes waveguide and material losses negligible. The S-band is used to avoid Fabry-Perot interference.

Due to the large working distance of the GRIN-lensed fiber of $1.9 \mathrm{~mm}$ and its wide lens radius of $0.25 \mathrm{~mm}$, the lens obscured the view on the test structures and an identification of the corresponding grating coupler proved to be difficult. In addition, the wavelength of $1550 \mathrm{~nm}$ does not lay in the visible range, hence conventional coupling, where the fiber is in direct contact with the grating, was not possible. As a consequence, to find the $x y$-position of the spot emitted from the GRIN-lensed fiber, a pilot laser was introduced in the optical path. Visible light from the HeNe pilot laser $(\lambda=632.816 \mathrm{~nm})$ was projected through the fiber onto the input grating coupler of the chip, as pointed out in Fig. 4 (top view). The proper working distance was set by measuring the pixels on the live image of the side camera 4 (side view), after a length calibration has been performed in software. At this point, the optical coupling was optimized and an optical power reference was recorded, from which we calculated the transmission for subsequent measurements.

The input fiber was then gradually moved by a high-precision three-axis translation stage in $1 \mu \mathrm{m}$ increments, where the zero position is meant to be the optimal coupling position. This resulted in a mapping of the transmission along all three axes $(x, y, z$ in a left-handed coordinate system), which is presented in Figs. 5-7.

As a figure of merit, we use the half-power bandwidth, that indicates the value at which the power has dropped by half. Along the $x$ axis (Fig. 5) the 3-dB bandwidth increases by almost six times, from 6 to $34 \mu \mathrm{m}$. Furthermore, the transmission profile shows a plateau with very little falloff between 0 and $20 \mu \mathrm{m}$. Here, the fiber position shifts from left to right and perpendicular to the grating, which explains the linear behavior around the plateau. A linear fit within this interval results in a slope of $\Delta T / \Delta x=-0.042 \mathrm{~dB} / \mu \mathrm{m}$.

Along the $y$ axis (Fig. 6), the 3-dB-bandwidth increases by more than three times, from 6 to $21 \mu \mathrm{m}$. The shift follows from back to forth and parallel to the grating. The polarization of the light is also parallel to the grating (TE mode). When fitted with a quadratic function, we deduce an expression for the transmission $T$ depending on the $y$ position

$$
T(y)=-0.0297 y^{2}-0.0729 y .
$$

On the $z$ axis (Fig. 7), the 3-dB bandwidth is increased by more than four times, from $19 \mu \mathrm{m}$ to $>80 \mu \mathrm{m}$. The shift follows from down to up. Due to the fact, that the SMF is virtually in direct contact with the chip surface $(w \approx 0 \mathrm{~mm})$, the value for the upward shift in reality improves by

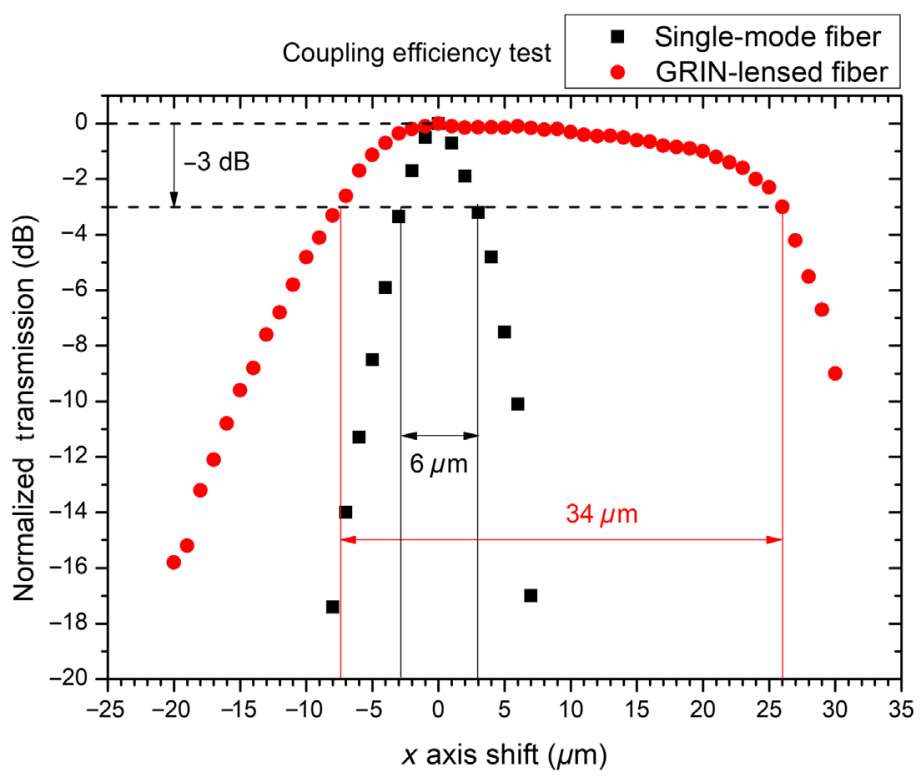

Fig. 5 Comparison of an SMF to the GRIN-lensed fiber toward the coupling efficiency along the $x$ axis. The $3-\mathrm{dB}$ bandwidth improves from 6 to $34 \mu \mathrm{m}$. 


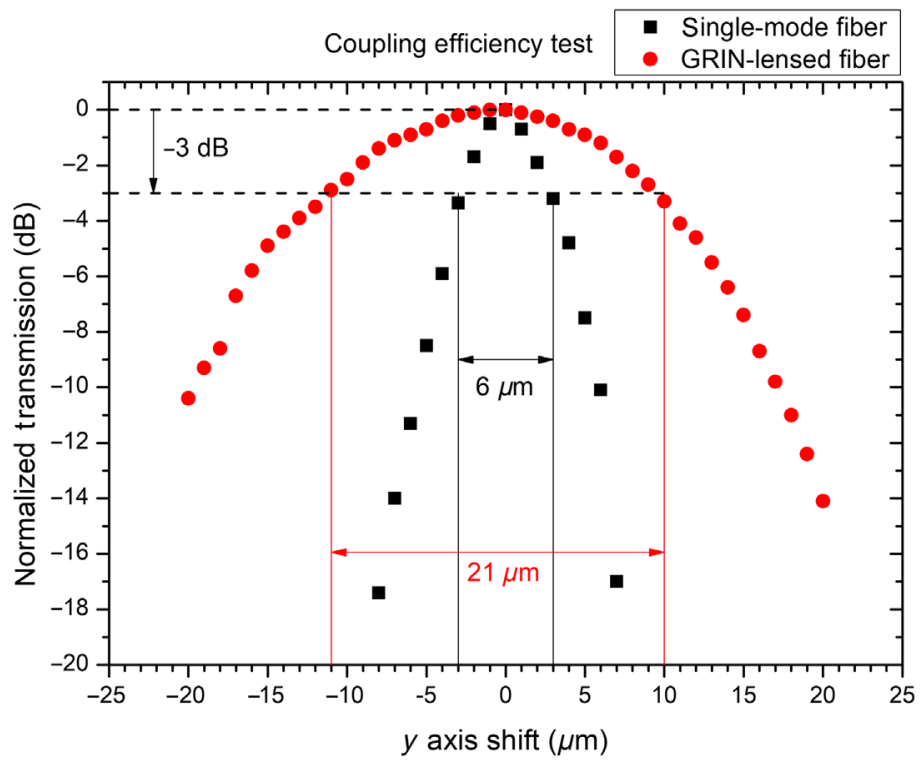

Fig. 6 Comparison of an SMF to the GRIN-lensed fiber toward the coupling efficiency along the $y$ axis. The 3-dB bandwidth improves from 6 to $21 \mu \mathrm{m}$.

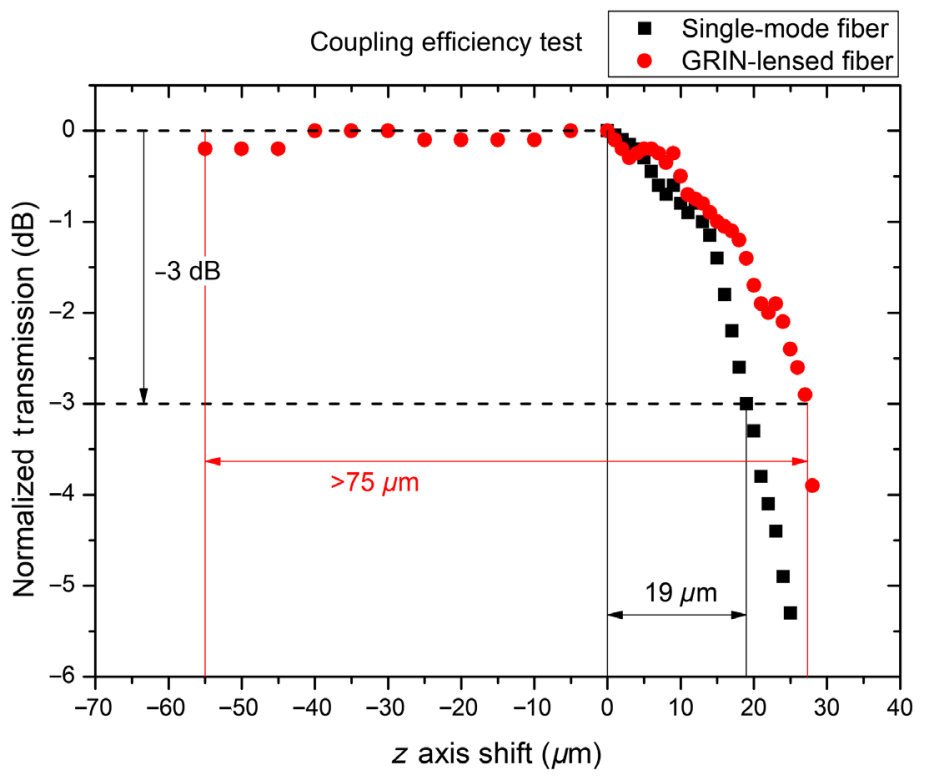

Fig. 7 Comparison of an SMF and the GRIN-lensed fiber toward the coupling efficiency along the $z$ axis. The 3-dB bandwidth improves from $19 \mu \mathrm{m}$ to $>80 \mu \mathrm{m}$. It is noteworthy that the SMF has a working distance close to $0 \mathrm{~mm}$, whereas the GRIN-lensed fiber can move down from its working distance of $1.9 \mathrm{~mm}$.

just $\approx 8 \mu \mathrm{m}$. When the GRIN-lensed fiber is moved down by $55 \mu \mathrm{m}$ from its working distance of $w=1.9 \mathrm{~mm}$, the spot size of the collimated light stays approximately constant over this short distance, therefore resulting in no change in the light transmission.

However, one drawback of the GRIN-lensed fiber for fiber-to-chip coupling is the increased optical losses mainly due to the fact that the grating coupler area is $150 \mu \mathrm{m}^{2}$ and the MFD of the fiber is $60 \mu \mathrm{m}$. At the peak of the transmission (zero position), the power is $7.8 \mathrm{~dB}$ lower than that of an SMF. This disadvantage could be overcome by simple increasing the optical power of the light source with, e.g., an amplifier. Additionally, anti-reflective coatings and GRINlenses with angled faces that reduce further reflections could increase the transmission. 
Table 3 Summary of the coupling test, comparing the efficiency of an SMF to a GRIN-lensed fiber at the input grating of the chip.

\begin{tabular}{lcccc}
\hline \hline & \multicolumn{3}{c}{ 3-dB bandwidth $(\mu \mathrm{m})$} & \\
\cline { 2 - 4 } Fiber type & $x$ axis & $y$ axis & $z$ axis & $\begin{array}{c}\text { Peak transmission } \\
(\mathrm{dBm})\end{array}$ \\
\hline SMF & 6 & 6 & 19 & -18.3 \\
GRIN-lensed fiber & 34 & 21 & $>80$ & -26.1 \\
\hline \hline
\end{tabular}

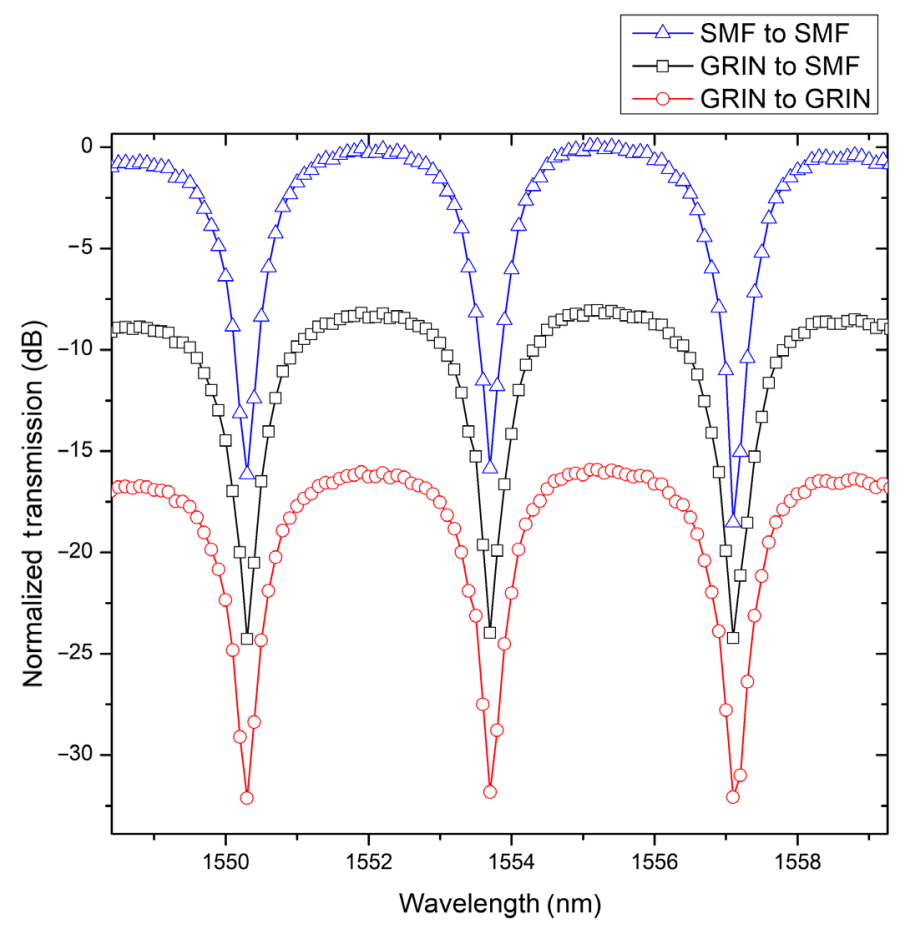

Fig. 8 Optical spectrum of a micro-ring resonator observed with different fibers as input and output fiber. Here, SMF to SMF, GRIN-lensed fiber to SMF (GRIN to SMF), and GRIN-lensed fiber to GRIN-lensed fiber (GRIN to GRIN) were measured. The input power of the tunable laser is $3 \mathrm{dBm}$.

The manufacturer specifies a reflection loss of $12 \%$ for the uncoated GRIN-lens. Table 3 gives a summary of the results from the coupling efficiency test. We can note not only the $x, y$, and $z$ axis have an influence on the coupling efficiency but also the coupling angle. However, the investigation of the coupling angle influence is not trivial. Changing the angle with the employed goniometer requires a readjustment of all other parameters $(x, y$, and $z$ axis).

Finally, we have tested the feasibility to measure the spectrum of a photonic sensor device with the proposed GRIN-lensed fiber. In this study, we measured the spectrum of a micro-ring resonator in the optical C-band. Micro-ring resonators are widely used as photonic label-free biosensors, ${ }^{9}$ thermal sensors, ${ }^{24}$ and electro-optical modulators. ${ }^{25}$ We employed the same experimental setup as described above. Figure 8 shows the observed optical spectra ranging from 1548.41 to $1559.26 \mathrm{~nm}$, indicating the feasibility of the GRIN-lensed fiber coupling technique by using an input power of $3 \mathrm{dBm}$.

\section{Conclusions}

In this work, we presented a comparative analysis of fiber-to-chip coupling tolerances using a GRIN-lensed fiber and an SMF. The presented results give an idea about the expected maximum 
coupling efficiency and alignment tolerance for coupling from a GRIN fiber collimator to a silicon waveguide. In particular, our experiments showed a 5.7 times higher alignment tolerance by using a GRIN-lensed fiber instead of an SMF. The lower peak transmission is sufficient to measure the optical spectrum of integrated micro-ring resonators, as we have proven by using a rather low laser input power of $3 \mathrm{dBm}$. The investigated approach is a promising candidate for future integrated photonic biosensors based on micro-ring resonators and the improved alignment tolerance gives rise to a pluggable photonic sensor solution.

\section{Acknowledgments}

This work has received funding from the ATTRACT project funded by the EC under Grant Agreement No. 777222. Parts of this work is funded by European Regional Development Fund (Grant No. 10.13039/501100008530).

\section{References}

1. X. Zou et al., "A multifunctional photonic integrated circuit for diverse microwave signal generation, transmission, and processing," Laser Photonics Rev. 13(6), 1800240 (2019).

2. M. L. Davenport et al., "Heterogeneous silicon/III-V semiconductor optical amplifiers," IEEE J. Sel. Top. Quantum Electron. 22(6), 78-88 (2016).

3. P. Steglich et al., "Quadratic electro-optical silicon-organic hybrid RF modulator in a photonic integrated circuit technology," in IEEE Int. Electron Dev. Meeting, pp. 23.3.1-23.3.4 (2018).

4. B. Wang et al., "A low-voltage $\mathrm{Si}-\mathrm{Ge}$ avalanche photodiode for high-speed and energy efficient silicon photonic links," J. Lightwave Technol. 38, 3156-3163 (2020).

5. P. Steglich et al., "Hybrid-waveguide ring resonator for biochemical sensing," IEEE Sens. J. 17(15), 4781-4790 (2017).

6. C. Qiu et al., "Demonstration of reconfigurable electro-optical logic with silicon photonic integrated circuits," Opt. Lett. 37(19), 3942-3944 (2012).

7. P. Steglich et al., "CMOS-compatible silicon photonic sensor for refractive index sensing using local back-side release," IEEE Photonics Technol. Lett. 32(19), 1241-1244 (2020).

8. P. Steglich et al., "Optical biosensors based on silicon-on-insulator ring resonators: a review," Molecules 24(3), 519 (2019).

9. E. Luan et al., "Silicon photonic biosensors using label-free detection," Sensors 18(10), 3519 (2018).

10. R. Marchetti et al., "Coupling strategies for silicon photonics integrated chips," Photonics Res. 7(2), 201-239 (2019).

11. D. Taillaert et al., "An out-of-plane grating coupler for efficient butt-coupling between compact planar waveguides and single-mode fibers," IEEE J. Quantum Electron. 38(7), 949-955 (2002).

12. D. Vermeulen et al., "High-efficiency fiber-to-chip grating couplers realized using an advanced CMOS-compatible silicon-on-insulator platform," Opt. Express 18(17), 1827818283 (2010).

13. G. Son et al., "High-efficiency broadband light coupling between optical fibers and photonic integrated circuits," Nanophotonics 7(12), 1845-1864 (2018).

14. A. Mekis et al., "A grating-coupler-enabled CMOS photonics platform," IEEE J. Sel. Top. Quantum Electron. 17(3), 597-608 (2011).

15. C. Scarcella et al., "Pluggable single-mode fiber-array-to-PIC coupling using micro-lenses," IEEE Photonics Technol. Lett. 29(22), 1943-1946 (2017).

16. P.-I. Dietrich et al., "In situ 3D nanoprinting of free-form coupling elements for hybrid photonic integration," Nat. Photonics 12(4), 241-247 (2018).

17. H. Melkonyan et al., "Gradient-index optical fiber lens for efficient fiber-to-chip coupling," Opt. Express 25, 13035-13045 (2017).

18. M. Zickar et al., "MEMS compatible micro-grin lenses for fiber to chip coupling of light," Opt. Express 14, 4237-4249 (2006). 
19. J. C. Palais, "Fiber coupling using graded-index rod lenses," Appl. Opt. 19, 2011-2018 (1980).

20. V. Vassiliev, S. Ilina, and V. Velichansky, "Diode laser coupled to a high-q microcavity via a grin lens," Appl. Phys. B 76(5), 521-523 (2003).

21. A. Joshi, S. Datta, and D. Becker, "Grin lens coupled top-illuminated highly linear ingaas photodiodes," IEEE Photonics Technol. Lett. 20(17), 1500-1502 (2008).

22. J. L. Coutaz and P. C. Jaussaud, "High index gradient in glass by ion exchange," Appl. Opt. 21, 1063-1065 (1982).

23. P. Steglich, C. Mai, and A. Mai, "Silicon-organic hybrid photonic devices in a photonic integrated circuit technology," ECS J. Solid State Sci. Technol. 8(11), Q217-Q221 (2019).

24. A. Mai et al., "Photonic thermal sensor integration towards electronic-photonic-IC technologies," in 49th Eur. Solid-State Dev. Res. Conf., pp. 254-257 (2019).

25. P. Steglich et al., "Electric field-induced linear electro-optic effect observed in siliconorganic hybrid ring resonator," IEEE Photonics Technol. Lett. 32(9), 526-529 (2020).

Siegfried Bondarenko received his master's degree in photonics from the Technische Hochschule Wildau in 2020. His current research is focused on photonic-integrated circuit technology for applications in telecommunication and biosensing.

Marcel Hülsemann received his master's degree in photonics from the Technische Hochschule Wildau in 2019. His current research is focused on microelectronic and optoelectronic packaging.

Andreas Mai received his diploma degree in physics and his $\mathrm{PhD}$ from the Technical University of Brandenburg. He joined the Process Integration Group, IHP Technology Department, Frankfurt (Oder), Germany, where he has acted as the Technology Department head since 2015. $\mathrm{He}$ is also a professor for micro- and nanoelectronics with Technische Hochschule Wildau, Wildau, Germany.

Patrick Steglich received his $\mathrm{PhD}$ from the Università degli Studi di Roma Tor Vergata, Rome, Italy, with a focus on integrated photonics for communication and sensing, in 2017. He is currently a scientist at IHP-Leibniz-Institut für innovative Mikroelektronik, Frankfurt, Germany, and lecturer for photonics and optical technologies at the TH Wildau. 